PROCEEDINGS OF THE

AMERICAN MATHEMATICAL SOCIETY

Volume 128, Number 5, Pages 1377-1380

S 0002-9939(99)05243-0

Article electronically published on August 17, 1999

\title{
A PERTURBED ERGODIC THEOREM
}

\author{
RADU-NICOLAE GOLOGAN
}

(Communicated by David R. Larson)

\begin{abstract}
Using a version of an ergodic lemma due to Cuculescu and Foiaş, we prove a pointwise ergodic theorem for $L^{1}$-contractions which can be viewed as a perturbed version of the celebrated ergodic theorem of Chacon and Ornstein. Surprisingly, to some extent, the complex part of the iterates involved have no effect on the ergodic convergence.
\end{abstract}

We shall recall some notations and results from [2] and 3]. Let $L^{1}=L^{1}(X, \mathcal{X}, \mu)$ be the usual complex $L^{1}$ space over a $\sigma$-finite measure space $(X, \mathcal{X}, \mu)$. By $T$ we shall denote a contraction acting in $L^{1}$. $T$ is called positive if the cone of positive functions, denoted by $L_{+}^{1}$, is $T$ - invariant. For $T$ an arbitrary contraction, we shall denote by $|T|$ the linear modulus of $T$, that is, the positive contraction satisfying $|T f| \leq|T| f$ for every $f \in L_{+}^{1}$ (see [5]). A sequence of positive measurable functions $\left(p_{n}\right)_{n \in \mathbb{N}}$ will be called $T$-adapted if for every $f \in L^{1}$ the inequalities $|f| \leq p_{n}$ for all $n \in \mathbb{N}$ imply $|T f| \leq p_{n+1}$ for every $n \in \mathbb{N}$.

Let $T^{*}$ denote the adjoint of $T$ acting on $L^{\infty}$. For $T$ a positive contraction and $A$ a measurable set, the equillibrium potential of $T$, denoted by $e_{A}$, is by definition the $L^{\infty}$-positive minimal function that satisfies $T^{*} e_{A} \leq e_{A}$ and $e_{A} \geq \chi_{A}$, where $\chi_{A}$ is the characteristic function of $A$ (see [3]). $\Re f$ will stand for the real part of the function $f$ and $f^{+}$(resp. $f^{-}$) for the positive part (resp. the negative part) of $\Re f$.

An easy consequence of the Maximal Ergodic Lemma of Cuculescu and Foias (3]) is the following result (see [4):

Maximal Ergodic Lemma. Suppose that $T$ is a positive contraction in $L^{1}$, $\left(g_{n}\right)_{n \in \mathbb{N}}$ a sequence of real $L^{1}$ functions and $A$ a measurable set such that:

$$
A \subset\left\{x \mid \sup _{n \geq k} \sum_{i=k}^{n} g_{i}(x)>0, \text { for every } k \geq 0\right\} .
$$

Then:

$$
\int\left[g_{0} e_{A}+\sum_{i=1}^{\infty}\left(g_{i}-T g_{i-1}\right)^{+} e_{A}\right] d \mu \geq \int\left[\sum_{i=1}^{\infty}\left(g_{i}-T g_{i-1}\right)^{-} e_{A}\right] d \mu .
$$

The proof of the following result is essentially based on the preceding lemma.

Received by the editors June 23, 1998.

1991 Mathematics Subject Classification. Primary 47A35, 28D99.

Key words and phrases. $L^{1}$-contraction, ergodic theorem.

The author was partially supported by the Romanian Academy, grant GAR 6645 .

(C)2000 American Mathematical Society 
Theorem. Suppose that $T$ is an arbitrary contraction in $L^{1},\left(p_{n}\right)_{n \in \mathbb{N}}$ a T-adapted sequence and $\left(q_{n}\right)_{n \in \mathbb{N}}$ a sequence of positive $L^{1}$-functions that satisfy:

$$
\int \sum_{i=1}^{\infty}\left(q_{i}-T q_{i-1}\right)^{+} d \mu<\infty
$$

Then:

$$
\lim _{n \rightarrow \infty} \frac{q_{0}+q_{1}+\cdots+q_{n}}{p_{0}+p_{1}+\cdots+p_{n}}
$$

exists and is finite $\mu$-a.e. on the set $\left\{x \mid \sum_{i=0}^{\infty} p_{i}(x)>0\right\}$.

Proof. A simple known trick easily reduces the proof to the case when $\left(p_{n}\right)_{n \in \mathbb{N}}$ is a sequence of $L_{+}^{1}$ functions such that $p_{n+1} \geq|T| p_{n}$, for $n \in \mathbb{N}$ (see [3]), so that in what follows we shall assume the latter condition fulfilled.

Observe also that $\left(q_{i}-T q_{i-1}\right)^{+}=\left(q_{i}-\Re T q_{i-1}\right)^{+} \geq\left(q_{i}-|T| q_{i-1}\right)^{+}$, for every $i \geq 1$, the latter being a consequence of the inequality $\Re T q_{i-1} \leq\left|T q_{i-1}\right| \leq|T| q_{i-1}$. Thus it will be sufficient to give the proof in the case that $T$ is a positive contraction.

It is also easily seen that in order to prove the convergence of the given ergodic ratio on the set $\left\{x \mid \sum_{i=0}^{\infty} p_{i}>0\right\}$ it will be suficient to prove it on the set $E_{0}=$ $\left\{x \mid p_{0}>0\right\}$.

For the reason of simplicity the following notation will be used:

$$
r_{n}=\frac{q_{0}+q_{1}+\cdots+q_{n}}{p_{0}+p_{1}+\cdots+q_{n}}
$$

where, as it was precised, $T$ is a positive contraction, $\left(p_{n}\right)_{n \in \mathbf{N}}$ satisfies $T p_{n} \leq p_{n+1}$ and we shall concentrate on the set $E_{0}$.

We shall first prove that $\limsup _{n \rightarrow \infty} r_{n}$ is finite a.e. on the set $E_{0}$ where $p_{0}>0$. For if not, there will be a measurable set $A \subset E_{0}, \mu(A)>0$ such that for all $\alpha>0$ the following will hold for any $k \in \mathbf{N}$ :

$$
\sup _{n \geq k} \sum_{i=k}^{n}\left(q_{i}-\alpha p_{i}\right)>0 \text {. }
$$

Denoting by $g_{i}=q_{i}-\alpha p_{i}$, the Maximal Ergodic Lemma will imply:

$$
\int\left[g_{0} e_{A}+\sum_{i=1}^{\infty}\left(g_{i}-T g_{i-1}\right)^{+} e_{A}\right] d \mu \geq \int \sum_{i=1}^{\infty}\left(g_{i}-T g_{i-1}\right)^{-} e_{A} d \mu .
$$

As $\left(g_{i}-T g_{i-1}\right)^{+}=\left[q_{i}-T q_{i-1}-\alpha\left(p_{i}-T p_{i-1}\right)\right]^{+} \leq\left(q_{i}-T q_{i-1}\right)^{+}$by the condition imposed in the hypothesis, both integrals are finite. Observing that $g_{0}=q_{0}-\alpha p_{0}$ and making $\alpha$ tend to $\infty$, we obtain $\int e_{A} p_{0} d \mu=0$. As $e_{A} \geq \chi_{A}$ and $A \subset E_{0}$, the last equality simply implies $\mu(A)=0$.

The proof that the limit exists goes now in a standard way. It will be sufficient to show that for any $a, b \in \mathbb{R}^{+}$, say $a>b$, the set $A \subset E_{0}$ where

$$
\liminf _{n \rightarrow \infty} r_{n}<a<b<\limsup _{n \rightarrow \infty} r_{n}
$$

is a null set.

The last inequalities have as consequences that for any $k \in \mathbb{N}$ one has:

$$
\sup _{n \geq k} \sum_{i=k}^{n}\left(a p_{i}-q_{i}\right)>0
$$


and

$$
\sup _{n \geq k} \sum_{i=k}^{n}\left(q_{i}-b p_{i}\right)>0
$$

Denote by $g_{i}=a p_{i}-q_{i}$ and $h_{i}=q_{i}-b p_{i}$ for any $i \geq 0$. By the Maximal Ergodic Lemma:

$$
\begin{gathered}
\int\left[h_{0} e_{A}+\sum_{i=1}^{\infty}\left(h_{i}-T h_{i-1}\right)^{+} e_{A}\right] d \mu \geq \int \sum_{i=1}^{\infty}\left(h_{i}-T h_{i-1}\right)^{-} e_{A} d \mu, \\
\int\left[g_{0} e_{A}+\sum_{i=1}^{\infty}\left(g_{i}-T g_{i-1}\right)^{+} e_{A}\right] d \mu \geq \int \sum_{i=1}^{\infty}\left(g_{i}-T g_{i-1}\right)^{-} e_{A} d \mu .
\end{gathered}
$$

As $\left(h_{i}-T h_{i-1}\right)^{+} \leq\left(q_{i}-T q_{i-1}\right)^{+}$, both integrals in the first inequality are finite. Thus:

$$
\int\left(q_{0}-b p_{0}\right) e_{A}+\sum_{i=1}^{\infty}\left(h_{i}-T h_{i-1}\right) e_{A} d \mu \geq 0
$$

the integral being finite. As $p_{i} \geq T p_{i-1}$ and $h_{i}-T h_{i-1}=\left(q_{i}-T q_{i-1}\right)^{+}-$ $\left(q_{i}-T q_{i-1}\right)^{-}-b\left(p_{i}-T p_{i-1}\right)$ we infer that the functions:

$$
\sum_{i=1}^{\infty}\left(q_{i}-T q_{i-1}\right)^{-} e_{A}
$$

and

$$
\sum_{i=1}^{\infty}\left(p_{i}-T p_{i-1}\right) e_{A}
$$

are integrable, implying that $\sum_{i=1}^{\infty}\left(g_{i}-T g_{i-1}\right) e_{A}$ is integrable. As a consequence the integrals in the second maximal inequality are finite and one can write:

$$
\int\left(a p_{0}-q_{0}\right) e_{A}+\sum_{i=1}^{\infty}\left(g_{i}-T g_{i-1}\right) e_{A} d \mu \geq 0
$$

where the left quantity is finite. Adding the relations (1) and (2) we infer:

$$
(a-b) \int\left[p_{0} e_{A}+\sum_{i=1}^{\infty}\left(p_{i}-T p_{i-1}\right) e_{A}\right] d \mu \geq 0 .
$$

As $a-b<0$ and $p_{i}-T p_{i-1} \geq 0$, we must have $e_{A}=0 \mu$-a.e. on $E_{0}$ and as $e_{A} \geq \chi_{A}$, we obtain $\mu(A)=0$.

Example. Consider a measure theoretical dynamical system on $X$, given by a measure preserving map $\phi: X \rightarrow X$. The classical ergodic theorem of Birkhoff asserts that for any measurable set $A \subset X$ the frequency that an orbit $\left(\phi^{n} x\right)_{n \in \mathbb{N}}$ enters $A$ exists $\mu$-a.e. and is $\mu(A)$ iff $\phi$ is ergodic.

Suppose now that at each stage we perturb $A$, say to $A_{n}$, such that

$$
\sum \mu\left(A_{n} \backslash A_{n-1}\right)<\infty \text {. }
$$


Our result asserts that in this case the frequency of the relation $\phi^{n} x \in A_{n}$ exists also a.e. For, considering $p_{i}=1, q_{i}=\chi_{\phi^{-1}} A_{i}$, and $\mathrm{T}$ the isometry induced by $\phi$, we have that

$$
\int\left(q_{i}-T q_{i-1}\right)^{+} d \mu=\int\left(\chi_{A_{i}}-\chi_{A_{i-1}}\right)^{+} d \mu=\mu\left(A_{i} \backslash A_{i-1}\right) .
$$

Remarks. It will be interesting to identify the limit in the theorem in terms of some measures defined using the sequences $\left(p_{n}\right)_{n \in \mathbb{N}},\left(q_{n}\right)_{n \in \mathbb{N}}$ in analogy with that in [4].

At this time we were not able to find similar conditions that imply the a.e.convergence if $\left(q_{n}\right)_{n \in \mathbb{N}}$ is a sequence of complex functions.

\section{REFERENCES}

[1] R.V. Chacon: Convergence of Operator Averages, Proc.Internat. Sympos. Ergodic Theory, Academic Press, New York 1963, 89-120. MR 28:4081

[2] R.V. Chacon, D.S. Ornstein: A general ergodic theorem, Ill. J. Math., 4, 1960, pp. 153-160. MR 22:1822

[3] C. Cuculescu, C. Foias: An individual ergodic theorem for positive operators, Rev. Roum. Math. Pures et Appl., Tome XI, 1966, pp. 581-594. MR 33:7495

[4] R.-N. Gologan: An extension of Chacon-Ornstein ergodic theorem, in Invariant Subspaces and other Topics, Birkhäuser Verlag, 1982, pp. 75-80. MR 84m:47014

[5] U.KRengel: Ergodic Theorems, Walter de Gruyter, Berlin; New York, 1985. MR 87i:28001

Institutul de Matematică Al Academiei Române, CP 1-764, 70700 Bucureşti, România

E-mail address: rgologan@stoilow.imar.ro 